\section{COVID-19 treatment in the older people: a brief clinical review}

\author{
Giovanni Cenderello, ${ }^{1}$ Simone Dini, ${ }^{2}$ \\ Serena Ziliani, ${ }^{3}$ \\ Crocefissa Fiorella Casuccio, ${ }^{4}$ \\ Francesca Mori Ubaldini ${ }^{1}$ \\ ${ }^{1}$ Infectious Diseases Department, ASL-1 \\ Imperiese, Sanremo; ${ }^{2}$ Geriatrics Unit, \\ Department Geriatric Care, \\ Orthogeriatrics and Rehabilitation, EO \\ Ospedali Galliera, Genova; ${ }^{3}$ Oncology \\ Department ASL1 Imperiese, Sanremo; \\ ${ }^{4}$ SC Medicina Interna, EO Ospedali \\ Galliera, Genova, Italy
}

\begin{abstract}
This paper summarizes the latest data about the treatment options available for coronavirus disease 2019 (COVID-19), paying special attention to the older patients. This review explored both trials that have included older patients and some clinically relevant drug-drug interactions. The data have been updated to $31^{\text {st }}$ October, 2020. In particular, details have been reported about the use of antivirals, heparin, steroid and hyperimmune plasma.
\end{abstract}

\section{Introduction}

Coronavirus disease 2019 (COVID-19) was initially observed in China in December 2019; it spread rapidly and was declared a pandemic by the World Health Organization (WHO) in March 2020. The disease is caused by the severe acute respiratory syndrome coronavirus 2 (SARS-CoV-2). ${ }^{1}$ The main symptoms consist of fever, acute respiratory failure mainly related to the interstitial pneumonia, with a potential involvement of many organs and functional discomfort. Indeed, COVID-19 may be considered a systemic disease in which every organ can be involved with different potential complications, such as ischemic, thrombo-embolic, and neuropathic complications. However, the most worrying clinical picture consists of an interstitial pneumonia with fast evolution, acute respiratory failure, and severe inflammatory reaction. COVID-19 related disease becomes an urgent problem due to the lack of proven, targeted treatments and the high infectivity and mortality rates, especially among frail older patients with pre-existing comorbidities, such as cardiac ischemia, hypertension, diabetes mellitus, or chronic obstructive pulmonary disease. ${ }^{2,3}$ As described by several authors, the mortality rate observed is age related, people older than 65 years present an odds ratio of 9.87 for death. ${ }^{4,5}$ Recent data produced by the Italian Institute of Health (ISS) has revealed a growing odds ratio for death with increasing age: $3.3 \%$ for subjects with an age over 60 years; $11.4 \%$ for subjects aged $\geq 70$ years; $20.3 \%$ for subjects aged $\geq 80$ years and $23.7 \%$ for subjects aged $\geq 90$ years (Table 1$).{ }^{6}$

Furthermore, recent studies reported a significant role of frailty, especially in older patients, to predict mortality and other negative health outcomes in patients with COVID19, suggesting the importance of frailty assessment for severity evaluation and decision-making processes..$^{7-9}$

The management of older frail patients is challenging not only due to the poor knowledge of the disease trajectories in old age, the high risk of disease progression towards a severe respiratory and multi-organ failures observed in older people but also for the long-term impact of the COVID-19 disease on functional, cognitive and psychosocial aspects of the older people. ${ }^{1}$ For all these reasons, a multidimensional approach to frailty, as consolidated in geriatric medicine, should be the best clinical management for the older patients with COVID-19. Indeed, a multidimensional approach for the management of the older patient with COVID-19 infection must take into account several factors related to frailty. ${ }^{11}$

It has been well known that the aging process is characterized by the biological phenomenon of inflamm-aging and immunosenescence. ${ }^{7}$ As a result of lifelong immunogenic stimulation, the old subject may have alterations of the adaptive immune system and the appearance of chronic inflammation. The subject is thus predisposed to contracting COVID-19 and developing a higher viral replication, lung tissue inflammation or, worse, injuries. ${ }^{12-14}$

Polypharmacotherapy is another important aspect to evaluate in the old patient. In particular, due to the increased risk of drug interactions and side effects due to polypharmacotherapy, great attention should be paid to the kind and the number of drugs to use for the treatment of COVID19 in the older patients.

Another aspect is the home management of the disease. This is also crucial, yet all supports are limited by the imposed isolation regimen. At the same time an early discharge from the hospital is a key to recovery especially in patient with multiple disabilities.

Finally, the disease treatment and supportive care are limited by the lack of ther-
Correspondence: Giovanni Cenderello, SC Malattie Infettive ASL-1 Imperiese, Via G. Borea 56, 18038 Sanremo, Italy.

Tel.: +39.0184536845 - Fax:+39.0184536840.

E-mail: g.cenderello@asl1.liguria.it

Key words: COVID-19 treatment; older people; aging; drug-drug interactions.

Acknowledgments: the authors are deeply grateful to Ms. Monica Valento for the language revision.

Contributions: SD, SZ and CFC searched the bibliography and performed the first analysis; SD, FMU and GC refined the first draft and modelled the final paper. All authors revised and approved the paper.

Received for publication: 30 August 2020.

Revision received: 11 December 2020.

Accepted for publication: 11 December 2020.

This work is licensed under a Creative Commons Attribution-NonCommercial 4.0 International License (CC BY-NC 4.0).

${ }^{\circ}$ Copyright: the Author(s), 2020

Licensee PAGEPress, Italy

Geriatric Care 2020; 6:9330

doi:10.4081/gc.2020.9330

apeutic protocols, especially for the frail, old patient. All these aspects are the basis of multidimensional geriatric approach and the best way to manage old frail patients. For this reason, and considering the previously reported diffusion of COVID-19 among older population, the creation of more new studies regarding multidimensional management of COVID-19 becomes more and more necessary. During COVID-19 outbreak, different drugs have been tested and the recommendations on therapy have rapidly changed in the last months (Table 2).

\section{Chloroquine and hydroxychloroquine}

The use of these drugs engendered considerable debate; in fact several papers reported deeply different results about efficacy and safety. From the beginning of the outbreak, preliminary data suggested that these drugs may play an important role in the management of COVID-19. ${ }^{15}$ They are known to have effects on the viral entry into host cells, and the immunomodulatory effect of hydroxychloroquine (HCQ) may help in controlling the cytokine storm. ${ }^{15}$ The Food and Drug Administration (FDA) authorized an unapproved indication due to 
the urgency of the situation. ${ }^{16}$ Both drugs are inexpensive and safe but can cause gastrointestinal intolerance and risk of QT interval (QTC) prolongation leading to potential lethal arrhythmia (Torsade de pointe or ventricular fibrillation), especially considering the possible presence of hypokalaemia and their frequent co-administration with azithromycin and/or analgesics and/or anti-psychotics.

Another important contraindication is the presence of glucose-6-phosphate dehydrogenase (G6PDH) deficiency which could be a limiting factor in the Mediterranean area. HCQ was found to be more potent than chloroquine. Several dosages were administered in clinical studies, and the best dosage seems to be 400 $\mathrm{mg}$ taken orally twice on the first day, followed by $200 \mathrm{mg}$ twice a day. ${ }^{17}$ Several drugs, such as azithromycin, anti-epileptics, and amiodarone, are involved in drug interactions, increasing the chloroquine dosage.

However, after the initial promising data, the WHO suspended the off-label use of these drugs. This decision was based on the results of two different trials where the use of HCQ was not associated with a reduction in mortality or in progression to mechanical ventilation in patients affected by COVID-19 pneumonia. ${ }^{18,19}$ The main concerns about the use of HCQ in older people are QTC prolongation and the addictive effect when used with the drugs largely used in geriatric care. The large majority of trials with HCQ have been conducted in Europe or USA in young people (the mean age of patients included in the cited French study cited was 43.6 years) so they were not presenting relevant comorbidities or risk of drug-drug interactions.

A single-center experience has been published regarding the safety issue, confirming the main risks. ${ }^{20}$

\section{Protease inhibitors (lopinavir-ritonavir or darunavir)}

A potential role for these drugs in COVID-19 management was hypothesized on the basis of limited virologic and clinical data on the use of lopinavir-ritonavir in SARS-CoV. ${ }^{21,22}$ Unfortunately, the test in vitro with darunavir did not show any activity for SARS-CoV-2 and was not able to prevent the infection in chronic HIV patients. ${ }^{23}$ Lopinavir-ritonavir was tested in a randomized trial, but the results did not show clinical improvement and $14 \%$ of patients could not complete the 14-day course due to adverse effects, such as gastrointestinal problems and QTC prolongation. ${ }^{24}$ Thus, the drugs belonging to this category were quickly withdrawn from treatment protocols. Tolerability was the main problem with this drug in older people.

\section{Remdesivir}

Remdesivir is a nucleotide analogue prodrug active against a broad spectrum of human and zoonotic coronaviruses. In fact, it is the only antiviral that has been confirmed to have a direct effect on SARS-CoV-2. Recently, it was authorized for compassionate use. In a study composed of 53 patients, clinical improvement in the oxygen support class was observed in $68 \%$ of patients. ${ }^{25}$ The improvement was more significant in patients younger than 50 years, but older patients (50-70 years and $>70$ years) reported significant improvement as well. ${ }^{25}$ However, it is worthy to underline that patients older than 70 years included in the study ${ }^{25}$ were just 18 , i.e. $34 \%$ of all patients enrolled in the

Table 1. Mortality rates (total, males, females) according to age (updated 18 November 2020) Italian Institute of Health. ${ }^{6}$

\begin{tabular}{|c|c|c|c|c|c|}
\hline Age class in years & $\begin{array}{c}\text { Male } \\
\text { (cases) }\end{array}$ & $\begin{array}{l}\text { Female } \\
\text { (cases) }\end{array}$ & $\begin{array}{l}\text { Total cases } \\
(\mathrm{M}+\mathrm{F})\end{array}$ & $\begin{array}{l}\% \text { death for class age } \\
\qquad(\mathrm{M}+\mathrm{F})\end{array}$ & $\begin{array}{l}\text { Lethality }(\%) \\
\qquad(\mathrm{M}+\mathrm{F})\end{array}$ \\
\hline $0-9$ & 22.665 & 21.176 & 43.841 & 0 & 0 \\
\hline $10-19$ & 55.644 & 49.734 & 105.378 & 0 & 0 \\
\hline $20-29$ & 78.248 & 74.423 & 152.675 & 0 & 0 \\
\hline $30-39$ & 74.837 & 75.517 & 150.360 & 0.2 & 0.1 \\
\hline $40-49$ & 90.355 & 103.147 & 193.504 & 0.9 & 0.2 \\
\hline $50-59$ & 107.247 & 113.281 & 220.532 & 3.5 & 0.7 \\
\hline $60-69$ & 73.553 & 60.862 & 134.416 & 9.8 & 3.3 \\
\hline $70-79$ & 55.077 & 47.109 & 102.186 & 25.5 & 11.4 \\
\hline $80-89$ & 38.199 & 53.883 & 92.089 & 41.1 & 20.3 \\
\hline$>90$ & 8.219 & 28.047 & 36.266 & 18.8 & 23.7 \\
\hline Age not reported & 63 & 57 & 120 & 0 & 7.5 \\
\hline Total & 604.107 & 623.236 & 1.231 .367 & 99.8 & 3.7 \\
\hline
\end{tabular}

Table 2. Evolution in COVID-19 treatment.

\begin{tabular}{lcc}
\hline Drug & March 2020 & August 2020 \\
Cloroquine and hydroxycloroquine & Suggested & Not recommended \\
IL1-IL6R inhibitors & Suggested in off-label use & Only in clinical trials \\
\hline Remdesivir & Promising & Approved by EMA and FDA \\
Dexamethasone & Contraindicated & Approved by EMA and FDA \\
\hline Low molecular weight heparin & Promising & Approved by EMA and FDA \\
Hyperimmmune plasma & Promising & Only for patients enrolled clinical trials
\end{tabular}


trial, and that the main safety issues were: a creatinine clearance above $30 \mathrm{ml}$ per minute and serum levels of alanine aminotransferase (ALT) and aspartate aminotransferase (AST) less than five times the upper limit of the normal range.

Further studies supported the use of this drug, leading the FDA and the European Drug Agency (EMA) to approve its use. In particular, a study proposed an optimization of the treatment by using only five days of treatment for patients not requiring mechanical ventilation. ${ }^{26}$ In this study were enrolled patients with confirmed diagnosis of SARS $\mathrm{CoV}-2$ infection by polymerase chain reaction assay within 4 days before randomization and radiographic evidence of pulmonary infiltrates and oxygen saturation of $94 \%$ or less. Both studies showed its safety even in older patients. ${ }^{25,26}$ The absence of randomization and the sample size require further confirmation. Moreover, based on previous experiments on SARS-CoV, remdesivir seems effective only when administered in the early stage.

\section{Immunomodulators}

The extensive use of immunomodulators has been proposed since the beginning of the COVID-19 outbreak; inhibitors of interleukin-6 (tocilizumab) and inhibitors of interleukin-1 were largely used in clinical trials and field tests. ${ }^{27,28}$ The rationale for administering these drugs was the evidence, at autopsy, of increased alveolar exudate caused by aberrant host immune response and inflammatory cytokine storm, which probably impedes alveolar gas exchange. Thus, the answer was to test drugs that could inhibit the storm. However, severe immune-suppression could expose the patients to the secondary risks posed by opportunistic infections. The results observed do not address this concern; an experience from Italy reported a rapid sustained clinical improvement (referred to respiratory condition) in 77 pts $(77 \%)$, associated with a significant radiological amelioration in 61 pts. ${ }^{29}$ More recently a paper by Mussini et al. seems to confirm the same results. ${ }^{30}$

Conversely, a randomized clinical trial (COVACTA) funded by the tocilizumab manufacturer did not meet the statistical significance in terms of improving clinical status in patients affected by COVID-19 pneumonia (primary objective of the study) nor a reduction in the mortality rate after 4 weeks (secondary objective of the study). These data excluded, for the moment, this drug as treatment options. ${ }^{31}$

\section{Heparin}

Endothelial injury as a consequence of SARS-CoV-2 infection leads to a dysregulated host inflammatory response and activation of coagulation pathways. Macroand micro-vascular thrombosis may contribute to morbidity, organ failure, and death. This was extensively reported in literature focused on the effects of arterial and venous thromboembolic complications of COVID-19, including venous thromboembolism (VTE), ischemic stroke, acute coronary syndrome (ACS), and myocardial infarction (MI).32 A study performed on 388 COVID-19 patients admitted to the hospital (median age 66 years Q1-Q3; 5575 years), found that thromboembolic events occurred in 28 patients $(21 \%)$, half of whom were diagnosed in the first $24 \mathrm{~h}$ of hospitalization; 44 patients underwent an Rx imaging, which confirmed a VTE in 16 patients $(36 \%)$. Computed tomography pulmonary angiogram (CTPA) was performed on 30 subjects $(7.7 \%$ of total cases); in 10 patients (33\% of CTPA subjects) a pulmonary embolism was confirmed. Both ischemic stroke and ACS or MI were observed in $2.5 \%$ and $1.1 \%$ of patients, respectively. Moreover, overt diffuse intravascular coagulation (DIC) was observed in 8 patients $(2.2 \%)$. The Authors concluded that due to the high number of arterial and venous thromboembolic events in hospitalized patients, especially in the first $24 \mathrm{~h}$ of admission, as well as due the high number of positive VTE cases at imaging examinations, VTE diagnostic strategies and thromboembolic events needed to be urgently improved and thromboprophylaxis considered for patients with COVID-19. These were also discussed with the inpatients with COVID19. Thus, therapeutic anticoagulation with heparin may improve clinical outcomes in patients with COVID-19 through the antithrombotic, anti-inflammatory, and antiviral activities of heparins. The approach of using heparins at a prophylactic dose in patients affected by COVID-19 is safe and well aligned with international guidelines for preventing venous embolism in older people. ${ }^{33}$

However, presently no studies showed improvement in mortality rate or toward mechanical ventilation with heparin use. So this approach needs to be careful evaluated, and we suggest to evaluate each patient according to the "Hypertension $A$ bnormal renal and liver function Stroke Bleeding Labile INR Elderly Drugs or alcohol' (HAS-BLED) score.

\section{Steroids}

The John Hopkins clinical guidance treatment of COVID-19, ${ }^{34}$ published on 25th March and updated on $7^{\text {th }}$ April 2020, excluded this drug from the options available due to the risk of reduction of viral clearance. In June 2020, the results of the multicenter RECOVERY trial showed that COVID-19 patients requiring mechanical ventilation or oxygen without ventilation had significantly lower 28-day all-cause mortality when randomized to dexamethasone rather than to standard care ${ }^{35}$ however, mortality did not differ between the dexamethasone group and the usual-care group in people who did not need respiratory support for COVID-19. RECOVERY investigators noted that inflammatory organ injury may affect people with advanced COVID-19. Yet, the role of glucocorticoids in quelling this inflammation remains controversial.

The trial participants received care at one of the 176 National Health Service (NHS) organizations in the United Kingdom. They had to have clinically suspected or lab-confirmed SARS-CoV-2 infection. An initial requirement that patients had to be at least 18 years old was removed on May 9, 2020. The investigators randomized participants in a $2: 1$ ratio to receive standard care alone or with oral or intravenous dexamethasone at a dose of 6 mg once a day for up to 10 days or until hospital discharge, whichever came first. The primary objective concerned all-cause mortality within 28 days of randomization. The analysis involved 2104 people randomized to dexamethasone and 4321 randomized to standard care. Their ages averaged at 66.9 years in the dexamethasone group and 65.8 years in the control group. Two-thirds of both groups $(64 \%)$ were men and, in both groups, a median of 2 days had passed since hospital admission. Similar proportions in the dexamethasone and control groups did not receive oxygen support ( $24 \%$ and $24 \%$ ), received only oxygen support $(61 \%$ and $60 \%$ ), and received invasive mechanical ventilation $(15 \%$ and $16 \%)$. The same proportion in each group (56\%) had previous coexisting diseases. Twenty-eight days after randomization, people receiving dexamethasone had significantly lower 28-day allcause mortality than the standard-care group (22.9\% versus $25.7 \%$, age-adjusted rate ratio $[R R] 0.83,95 \%$ confidence interval $[\mathrm{CI}] 0.75$ to $0.93, \mathrm{P}<0.001)$. Among the participants requiring invasive mechanical ventilation, all-cause mortality was one third lower in the dexamethasone group (29.3\% versus $41.4 \%$, age-adjusted $\mathrm{RR}$ $0.64,95 \%$ CI 0.51 to 0.81 ). Among people 
who needed oxygen support without ventilation, mortality was about $20 \%$ lower in those receiving dexamethasone $(23.3 \%$ versus $26.2 \%$, age-adjusted RR $0.82,95 \%$ CI 0.72 to 0.94$)$. Age-adjusted absolute drops in 28-day all-cause mortality with dexamethasone were 12.3 percentage points $(95 \%$ CI 6.3 to 17.6) in people needing mechanical ventilation and 4.2 percentage points (95\% CI 1.4 to 6.7$)$ in people needing oxygen but not ventilation. Dexamethasone had no mortality advantage among people who did not need oxygen support or mechanical ventilation $(17.8 \%$ versus $14.0 \%$, ageadjusted RR $1.19,95 \%$ CI 0.91 to 1.55 ). The results were similar when the researchers limited the analysis to 5698 people $(89 \%)$ who were polymerase chain reaction (PCR)-positive for SARS-CoV-2. When compared with the control group, participants randomized to dexamethasone spent less time in the hospital (median 12 versus 13 days) and had a $10 \%$ higher chance of discharge within 28 days (RR $1.10,95 \%$ CI 1.03 to 1.17 ). The researchers observed a trend toward lower progression to a pre-specified composite outcome of invasive mechanical ventilation or death in the dexamethasone group (RR $0.92,95 \%$ CI 0.84 to 1.01 ). The risk of progression to mechanical ventilation was almost $25 \%$ lower in people randomized to dexamethasone (RR $0.77,95 \%$ CI 0.62 to 0.95 ). The RECOVERY team observed that their findings confirm the results of a previous multicenter Spanish trial on 277 people with acute respiratory distress syndrome requiring mechanical ventilation, which found $15 \%$ lower mortality at 60 days with dexamethasone versus usual care. ${ }^{36}$ The investigators also noted that RECOVERY enrolled about $15 \%$ of all in-hospital COVID-19 patients in the United Kingdom, and mortality in their standard-care group reflected overall mortality in hospitalized COVID-19 patients across the country.

To support these data, a recent metaanalysis concluded that even if the literature to date does not fully support the routine use of corticosteroids in COVID-19, but some findings suggest that methylprednisolone could lower mortality rate in more severe forms of the condition. ${ }^{37}$

\section{Convalescent plasma}

Convalescent plasma has been used to treat infectious diseases since the early 20th century and was associated with reduced mortality during the 1918 influenza, ${ }^{38}$ SARS,${ }^{39}$ and H1N1 pandemics. ${ }^{40}$

Some anecdotal studies reported its potential use in treating patients affected by COVID-19. ${ }^{41}$ Recently, a randomized trial in China ${ }^{42}$ did not result in a statistically significant improvement as compared to clinical improvement within 28 days. The trial was limited by the low number of patients enrolled - only 103 rather than the 200 planned - and thus, further studies are required to define the efficacy of this approach. The patients' median age median age of 60 years and the low incidence of side effects (only two patients over 52 experienced adverse effects after transfusion) make this treatment safe and promising for old and frail people.

\section{Conclusions}

At the time of this revision, only three treatments are recommended by the FDA and EMA; they are: i) remdesivir as a directly active antiviral that should be prescribed when COVID-19 pneumonia is diagnosed; ii) dexamethasone for patients with respiratory failure; and low molecular weight; iii) heparin to prevent embolic complications or treat pulmonary embolism. The clinical use of these treatments should be tailored on the single older patient according to a careful multidimensional assessment and management care plan.

\section{References}

1. World Health Organization. WHO Director-General's opening remarks at the media briefing on COVID-19; 11 March, 2020. Available from: https://www.who. $\mathrm{int} / \mathrm{dg} /$ speeches/detail/who-directorgeneral-s-opening-remarksat-the-media-b riefing-on-covid-19---11-march-2020 Last access: 20 August 2020.

2. European Commission. COVID-19 cases and case fatality rate by age; 4 March 2020. Available from: https://ec.europa. eu/knowledge4policy/publication/covid19-cases-case-fatality-rate-age en Last access: 20 August 2020.

3. Zhou F, Yu T, Du R, et al. Clinical course and risk factors for mortality of adult inpatients with COVID-19 in Wuhan, China: A retrospective cohort study. Lancet 2020;395:1054-62.

4. Li X, Xu S, Yu M, et al. Risk factors for severity and mortality in adult COVID19 inpatients in Wuhan. J Allergy Clin Immunol 2020;146:110-8.

5. Du RH, Liang LR, Yang CQ, et al. Predictors of mortality for patients with COVID-19 pneumonia caused by SARS-
CoV-2: A prospective cohort study. Eur Respir J 2020;55:2000524.

6. EpiCentro. Caratteristiche dei pazienti deceduti positivi all'infezione da SARSCoV-2 in Italia; 27 March 2020 - Last update: 12 December 2020. Available from: https://www.epicentro.iss.it/ coronavirus/sars-cov-2-decessi-italia

7. Bellelli G, Rebora P, Valsecchi MG, et al. Frailty index predicts poor outcome in COVID-19 patients. Intensive Care Med 2020;46:1634-36.

8. Fulop T, Witkowski JM, Olivieri F, Larbi A. The integration of inflammaging in age-related diseases. Semin Immunol 2018;40:17-35.

9. Hewitt J, Carter B, Vilches-Moraga A, et al. The effect of frailty on survival in patients with COVID-19 (COPE): a multicentre, European, observational cohort study. Lancet Public Health 2020; 5:e444-51.

10. Lithander FE, Neumann S, Tenison E, et al. Covid-19 in older people: a rapid clinical review. Age Ageing 2020;49: 505-15.

11. Pilotto A, Custodero C, Maggi S, et al. A multidimensional approach to frailty in older people. Ageing Res Rev 2020;60: 101047.

12. Velazquez-Salinas L, Verdugo-Rodriguez A, Rodriguez LL, Borca MV. The role of interleukin 6 during viral infections. Front Microbiol 2019;10:1057.

13. Maggio M, Guralnik JM, Longo DL, Ferrucci L. Interleukin-6 in aging and chronic disease: A magnificent pathway. J Gerontol A Biol Sci Med Sci 2006; 61:575-84.

14. Bonafè M, Prattichizzo F, Giuliani A, et al. Inflamm-aging: Why older men are the most susceptible to SARS-CoV-2 complicated outcomes. Cytokine Growth Factor Rev. 2020;53:33-7

15. Van den Borne BE. Chloroquine and hydroxychloroquine equally affect tumor necrosis factor-alpha, interleukin 6 , and interferon-gamma production by peripheral blood mononuclear cells. J Rheumatol 1997;24:55-60.

16. Food and Drug Administration; 2020. Available from: https://www.fda.gov/ media/136537/download Accessed: 15 April 2020.

17. Yao X, Ye F, Zhang M, Cui C. In vitro antiviral activity and projection of optimized dosing design of hydroxychloroquine for the treatment of severe acute respiratory syndrome coronavirus 2 (SARS-CoV-2). Clin Infect Dis 2020;71:732-9.

18. Mahévas M, Tran VT, Roumier M, et al. Clinical efficacy of hydroxychloroquine in patients with covid-19 pneumonia 
who require oxygen: observational comparative study using routine care data. BMJ 2020;369:m1844.

19. Geleris J, Sun Y, Platt J, et al. Observational study of hydroxychloroquine in hospitalized patients with covid-19. N Engl J Med 2020;382:2411-8.

20. Forni N, Boasi V, Sciole K, et al. QTc prolongation during the SARS-CoV-2 outbreak: clinical risk management through hospital training course, focus on elderly patients. Geriatric Care 2020;6: 9128.

21. Chu CM, Cheng VC, Hung IF, et al. Role of lopinavir/ritonavir in the treatment of SARS: initial virological and clinical findings. Thorax 2004;59:252-56.

22. Kim UJ, Won EJ, Kee SJ, et al. Combination therapy with lopinavir/ ritonavir, ribavirin and interferon- $\alpha$ for Middle East respiratory syndrome. Antivir Ther 2016;21:455-9.

23. Riva A, Conti F, Bernacchia D, et al. Darunavir does not prevent SARS-CoV2 infection in HIV patients. Pharmacol Res 2020;157:104826.

24. Cao B, Wang Y, Wen D, et al. A Trial of lopinavir-ritonavir in adults hospitalized with severe Covid-19. N Engl J Med 2020;382:1787-99.

25. Grein J, Ohmagari N, Shin D, et al. Compassionate use of remdesivir for patients with severe Covid-19. N Engl J Med 2020;382:2327-36.

26. Goldman JD, Lye DCB, Hui DS, et al. Remdesivir for 5 or 10 Days in Patients with Severe Covid-19. N Engl J Med 2020; NEJMoa2015301 [Epub ahead of print].

27. Zhang C, Wu Z, Li JW, et al. Cytokine release syndrome in severe COVID-19: Interleukin-6 receptor antagonist tocilizumab may be the key to reduce mortality. Int $\mathrm{J}$ Antimicrob Agents 2020;55:105954.

28. Pontali E, Volpi S, Antonucci G, et al. Safety and efficacy of early high-dose IV anakinra in severe COVID-19 lung disease. J Allergy Clin Immunol 2020;146:213-5.

29. Toniati P, Piva S, Cattalini M, et al. Tocilizumab for the treatment of severe COVID-19 pneumonia with hyperinflammatory syndrome and acute respiratory failure: A single center study of 100 patients in Brescia, Italy. Autoimmun Rev 2020;19:102568.

30. Guaraldi G, Meschiari M, Cozzi-Lepri A, et al. Tocilizumab in patients with severe COVID-19: a retrospective cohort study. Lancet Rheumatol 2020;2:e474-84.

31. Roche.com. Roche provides an update on the phase III COVACTA trial of Actemra/RoActemra in hospitalised patients with severe COVID-19 associated pneumonia. Available from: https://www.roche.com/media/releases/m ed-cor-2020-07-29.htm Last access: 20 August 2020.

32. Lodigiani C, Iapichino G, Carenzo L, et al. Venous and arterial thromboembolic complications in COVID-19 patients admitted to an academic hospital in Milan, Italy. Thromb Res 2020;191:9-14.

33. Robert-Ebadi H, Le Gal G, Righini M. Use of anticoagulants in elderly patients: practical recommendations. Clin Interv Aging 2009;4:165-77.

34. Writing Group of the Johns Hopkins University and Johns Hopkins Hospital COVID-19 Treatment Guidance Working Group. The JHMI Clinical Guidance for treatment of COVID-19; Update: 25 March 2020. Available from: https:// www.hopkinsguides.com/hopkins/ub?cm $\mathrm{d}=$ repview\&type $=479$ -

1125\&name $=4$ 538747_PDF_ Last access: 20 August 2020

35. RECOVERY Collaborative Group, Horby P, Lim WS, et al. Dexamethasone in Hospitalized Patients with Covid-19 Preliminary Report [published online ahead of print, 2020 Jul 17]. N Engl J Med 2020;NEJMoa2021436.

36. Villar J, Ferrando C, Martínez D, et al. Dexamethasone treatment for the acute respiratory distress syndrome: A multicentre, randomised controlled trial. Lancet Respir Med 2020;8:267-76.

37. Veronese N, Demurtas J, Yang L, et al. Use of corticosteroids in coronavirus disease 2019 pneumonia: a systematic review of the literature. Front Med 2020;7:170.

38. Luke TC, Kilbane EM, Jackson JL, Hoffman SL. Meta-analysis: convalescent blood products for Spanish influenza pneumonia: A future $\mathrm{H} 5 \mathrm{~N} 1$ treatment? Ann Intern Med 2006;145: 599-09.

39. Cheng Y, Wong R, Soo YO, et al. Use of convalescent plasma therapy in SARS patients in Hong Kong. Eur J Clin Microbiol Infect Dis 2005;24:44-6.

40. Hung IF, To KK, Lee CK, et al. Convalescent plasma treatment reduced mortality in patients with severe pandemic influenza A (H1N1) 2009 virus infection. Clin Infect Dis 2011;52:447-56.

41. Shen C, Wang Z, Zhao F, et al. Treatment of 5 critically ill patients with COVID-19 with convalescent plasma. JAMA 2020;323:1582-89.

42. Li L, Zhang W, Hu Y, et al. Effect of convalescent plasma therapy on time to clinical improvement in patients with severe and life-threatening COVID-19: a randomized clinical trial. JAMA 2020;324:460-70 [published correction appears in JAMA 2020;324:519]. 